\title{
Change in the Frequency of Seizure Attacks and Associated Factors Among Adult Epilepsy Patients at Amanuel Mental Specialized Hospital (AMSH): A Generalized Linear Mixed Model (GLMM)
}

\author{
Temam Beshir Raru (1D) \\ Bisrat Misganaw Geremew ${ }^{2}$ \\ Koku Sisay Tamirat $\mathbb{D}^{2}$ \\ 'Department of Epidemiology and \\ Biostatistics, School of Public Health, \\ Haramaya University, Harar, Ethiopia; \\ ${ }^{2}$ Department of Epidemiology and \\ Biostatistics, Institute of Public Health, \\ College of Medicine and Health Sciences, \\ University of Gondar, Gondar, Ethiopia
}

Correspondence: Temam Beshir Raru Email Temam.bI@gmail.com
Background: While getting anti-epileptic drugs (AEDs) treatment around two-thirds of the patients with epilepsy enter a seizure-free state, but some of them continue to experience seizures which increase the risk of accident, disability, death, and treatment side effects. Therefore, this study aimed to identifychanges in the frequency of seizure attacks overtime and associated factors among adult patients with epilepsy.

Methods: A retrospective two year follow-up study was conducted among adult patients with epilepsy who initiated AEDs between July 2017 and June 2019. A simple random sampling technique was applied to select 394 patient charts. The data were entered into Epidata and then exported to R software for further analysis. A generalized linear mixed model under a negative binomial family was applied to identify determinants of the frequency of seizure attacks.

Results: The median number of seizure attacks at baseline were 3 with the IQR of 2 and on average the frequency of seizure attacks decrease in a linear pattern over time. Age group 25-34 years $(\mathrm{AIRR}=0.81$ and 95\% CI: 0.69-0.94), smoking $(\mathrm{AIRR}=1.28$ and $95 \% \mathrm{CI}$ : 1.11-1.48), khat use (AIRR $=1.39$ and 95\% CI: 1.19-1.62), sleep deprivation (AIRR = 1.49 and $95 \% \mathrm{CI}: 1.32-1.69$ ), head injury (AIRR $=1.30$ and $95 \% \mathrm{CI}: 1.11-1.53$ ), depression $(\mathrm{AIRR}=1.39$ and $95 \% \mathrm{CI}: 1.17-1.65)$, treatment mode $(\mathrm{AIRR}=1.22$ and $95 \% \mathrm{CI}: 1.07-$ $1.39)$, and clumsiness $(A I R R=1.85$ and $95 \% C I: 1.24-2.75)$ were statistically significant seizure predicting factors.

Conclusion: The number of seizure attack changes over time was decreased by a linear function that might reflect treatment effects of AEDs. Smokers, khat chewers, suffering a head injury, depression, experienced sleep deprivation, and clumsiness increases the number of seizure attacks. Conversely, the age group 25-34 years saw a decrease in the number of seizure attacks. This underscores that special attention should be given to substance users and those with related mental health disorders.

Keywords: change in seizures frequency, epilepsy, anti-epileptic drugs, Ethiopia, AEDs

\section{Introduction}

Epilepsy is a chronic brain dysfunction characterized by an abnormal, excessive, and hyper-synchronous electrical discharge of neurons in the brain which results in the appearance of epileptic seizures. It may be partial which affects only one hemisphere of the brain, or generalized which affects the entire part of the brain. They may be accompanied by loss of consciousness and loss of control of bowel or bladder functions. 
Globally, there are 50 million people with epilepsy, and the majority (80\%), of whom live in low and middle-income countries (LMIC), where treatment gaps exceed $75 \%$ in most low-income countries and 50\% in most middle-income countries. Moreover, the prevalence of epilepsy in Africa ranges from 2.2 to 58 per 1000 population and this makes it the second or third most common reason for consultation and hospitalization. ${ }^{1,2}$ While getting AEDs treatment around two-thirds of patients with epilepsy enter a seizure-free state, but some may continue to experience any of the previously mentioned types of seizure. ${ }^{3}$

Epileptic patients have poor health outcomes, with greater psychological distress, depression, anxiety, employment restriction, physical injuries such as fractures and burns, and increased mortality. Epileptic seizures also result in devastating social consequences which can lead to a poor quality of life. ${ }^{4}$ Seizure frequency is a significant factor for depression which is among the leading causes of disability. When the number of seizure attacks increases, depression and perceived stigma also increases, which further results in devastating social consequences and diminished quality of life. ${ }^{5}$

A study conducted in Ethiopia shows that out of 194 participants, the majority, 171 (88.1\%), of the participants had generalized tonic-clonic seizures (GTCS) and the remaining had focal (partial) seizures ${ }^{6}$ Another retrospective study on the status epilepticus (SE) stated that from the total study participants who developed GTCS at baseline evaluation, with the frequency of seizure attacks $\leq 8$ times and 9-12 times per follow-up period, 23.2\% and $13.4 \%$ of them died, respectively, but there is no history of death among those who have $>13$ times frequency of seizure attacks. Among the participants with an unknown history of seizure frequency, $10.3 \%$ died due to several factors, but CNS infection and non-compliance with AED account for $43 \%$ and $21 \%$, respectively. ${ }^{7}$

Some patients may continue to experience seizures while taking anti-epileptic treatment, even if the uttermost goal of treating epileptic patients is to maintain a seizure-free state. Assessment of seizure frequency is done to show treatment response and is also helpful for stopping treatment, when an individual is seizure-free for at least two years the assessment can aid clinical decision-making. Hence, identifying seizurepredicting factors and changes in the frequency of seizure attacks over time is crucial to improve the patients' health status and quality of life.
Therefore, this study aims to fill the gaps of current knowledge about epilepsy treatment by identifying determinant risk factors of epilepsy and changes in the number of seizure attacks.

\section{Methods}

\section{Study Design and Period}

An institution-based retrospective two year follow-up study was conducted among adult epileptic patients who initiated treatment between July 2017 and June 2019.

\section{Study Area and Population}

The study was conducted at Amanuel Mental Specialized Hospital (AMSH) located in the western part of Addis Ababa in Addis Ketema Sub-city Kebele 08. It has been serving as the only public specialized psychiatric hospital since 1948 under the Ministry of Health. The hospital has 259 beds including 11 private wing beds and 23 emergency beds. The hospital has 13 outpatient departments. An average of 26,400 follow-up and new patients receive outpatient services annually, and most of the patients attend with family members. A total of 2200 patients with epilepsy are seen by the neuropsychiatry case team each month, and it ranks second in outpatient department patient volume in the hospital.

Adult patients of epilepsy on AED treatment follow-up between 1 July 2017 and 30 June 2019 in the hospital was the study population. Meanwhile, patients who initiated antiepileptic medications between July 2017 and June 2019 and had at least two measurements for the outcome of interest were included. Whereas, patients who had incomplete records for major variables like the outcome of interest and had no follow-up sheets within their medical records were excluded. In addition, those patients who had been diagnosed to have SE were also excluded.

\section{Sample Size and Sampling Procedure}

The sample size was calculated by using Diggle et al formula for sample size calculation of repeated measurements. ${ }^{8}$ The final sample size for this study was 394 with $95 \%$ CI and $90 \%$ power. Initially eligible study population who have enrolled in antiepileptic treatment since July 2017 was identified (filtered) from the hospital medical record unit or documentation unit. Then final study subjects were selected by a simple random sampling technique using computer-generated random numbers by 
$\mathrm{R}$ software and stopped when the calculated sample reached.

\section{Data Collection Procedures}

A data collection checklist was prepared and used to extract socio-demographic, behavioral, clinical, and treatment-related characteristics of patients by reviewing patient charts. Data for this study was secondary data routinely recorded from patients followed up at AMSH usually every month. BSc psychiatry nurses who have been working on neuropsychiatry (NEP) cases were assigned as data collectors.

\section{Measurements}

In this study, the outcome variable was the frequency of seizure attacks which is the number of uncontrolled involuntary shaking involving only one part of the brain or involving the entire brain. The independent variables were age, sex, marital status, educational status, occupation, head injury, burn, fracture, depression, psychotic disorder, anxiety, duration, drug type, type of seizure, and treatment modalities.

The frequency of the seizure attacks was registered by asking either the patients or family members, or relatives in the vicinity of a patient since GTCC and focal seizures with loss of consciousness are usually cannot be observed by the patient himself.

Khat: is a stimulant drug, which speeds up the transmission of impulses between the brain and the body. ${ }^{9}$

\section{Ethics Approval and Consent to Participate}

The ethical review committee of the Institute of Public Health, College of Medicine and Health Sciences, University of Gondar approved the study protocol, and a letter of approval with Reference Number IPH/837/6/ 2020 was granted. A waiver letter was obtained from the medical director of the hospital to access the medical records of patients. Because data were collected from the patient's follow-up chart, informed consent was not obtained from all participants in the study. Confidentiality during all phases of research activities was kept and data was held on a secured passwordprotected system. All the procedures are based on the principles of the Helsinki declaration.

\section{Statistical Analysis}

The data were checked for inconsistencies, coding error, completeness, clarity, and missing values before it was entered. The data entry was done using Epi-data version 4.64.0 and then it was exported to $\mathrm{R}$ statistical software Version 3.5.2 for further analysis. Missing data were handled by using multiple imputations techniques.

Descriptive statistics were carried out and presented using tables, text, and graphs. Individual, mean profile plots and observed variance plots were considered as parts of the data exploration technique which gives some directions to select the appropriate statistical model to a given data.

Both bi-variable and multivariable generalized linear mixed models under a negative binomial family analysis were performed. To determine the association between the frequency of seizure attack and explanatory variables using a generalized linear mixed model and checked for over-dispersion and zero-inflation in the data using R-software. Variables with a p-value $<0.2$ in the bi-variable analysis were selected for multivariable analysis.

Akaike information criteria (AIC), Bayesian information criteria (BIC), and Deviance were applied for model selection after fitting the appropriate model for the data by assuming random intercept only model, the random slope only model, and random intercept and random slope model. Finally, the adjusted incidence rate ratio (AIRR) with $95 \%$ CI was presented and interpreted accordingly.

\section{Results}

\section{Socio-Demographic and Behavioral Characteristics}

A total of 394 patients' charts were reviewed and their ages ranged between 15 to 65 years. The average age of the participants was 25.94 with a standard deviation (SD) of 10.76 years. The majority of the participants $(56.35 \%)$ were aged $15-24$ years and $56.85 \%$ of the participants were male. Nearly two-thirds $(63.45 \%)$ of the participants were single and $13.71 \%$ had an educational status of diploma and above. Regarding their occupation, around $52.54 \%$ of the participants were students. A history of alcohol consumption was seen in $30.20 \%$ of the participants and only $22.10 \%$ of patients had a family history of epilepsy. Besides, $43.65 \%$ of them suffered from sleep deprivation, in which the seizures occurred after it (Table 1). 
Table I Socio-Demographic and Behavioral Characteristics of AED Attendants, Amanuel Mental Specialized Hospital, 2017-2019

\begin{tabular}{|c|c|}
\hline Variables with Category & $\begin{array}{l}\text { Epilepsy Cases Among Each } \\
\text { Category }(n=394)(\%)\end{array}$ \\
\hline \multicolumn{2}{|l|}{ Age at diagnosis } \\
\hline $15-24$ & $222(56.35)$ \\
\hline $25-34$ & $96(24.37)$ \\
\hline $35-44$ & $50(12.69)$ \\
\hline $45-54$ & $10(2.54)$ \\
\hline $55-65$ & $16(4.06)$ \\
\hline \multicolumn{2}{|l|}{ Sex } \\
\hline Male & $224(56.85)$ \\
\hline Female & $170(43.15)$ \\
\hline \multicolumn{2}{|l|}{ Marital Status } \\
\hline Single & $250(63.45)$ \\
\hline Married & $130(32.99)$ \\
\hline Divorced & $9(2.28)$ \\
\hline Widowed & $5(1.27)$ \\
\hline \multicolumn{2}{|l|}{ Occupation } \\
\hline Government Employed & $23(5.84)$ \\
\hline Non-government employed & $76(19.29)$ \\
\hline Farmer & $54(|3.7|)$ \\
\hline Student & $207(52.54)$ \\
\hline Housewife & $34(8.63)$ \\
\hline \multicolumn{2}{|l|}{ Educational Status } \\
\hline No formal education & $54(|3.7|)$ \\
\hline Primary & $117(29.70)$ \\
\hline Secondary & $169(42.89)$ \\
\hline Diploma and above & $54(|3.7|)$ \\
\hline \multicolumn{2}{|l|}{ Smoking } \\
\hline Yes & $79(20.05)$ \\
\hline No & $315(79.95)$ \\
\hline \multicolumn{2}{|l|}{ Alcohol } \\
\hline Yes & $119(30.20)$ \\
\hline No & $275(69.80)$ \\
\hline \multicolumn{2}{|l|}{ Khat } \\
\hline Yes & $7 I(18.02)$ \\
\hline No & $323(81.98)$ \\
\hline \multicolumn{2}{|l|}{ Sleep deprivation } \\
\hline Yes & $172(43.65)$ \\
\hline No & $122(56.35)$ \\
\hline \multicolumn{2}{|l|}{ Family History } \\
\hline Yes & $87(22.08)$ \\
\hline No & $307(77.92)$ \\
\hline
\end{tabular}

\section{Clinical and Treatment-Related}

\section{Characteristics}

Around $15.74 \%$ of participants had a history of head injury which is the result of epilepsy and $16.75 \%$ of them had a psychotic disorder, i.e., brief psychotic disorder. Nearly two-thirds $(64 \%)$ of patients had a generalized type of seizure attack and the mean duration of the illness was 5.06 years $(\mathrm{SD}=3.80)$. More than half $51.50 \%$ of the participants were treated by phenobarbital and mono treatment modality was seen in $74.40 \%$ of the patients. About $8.63 \%$ of the patients had complainted of treatment-related side effects, like clumsiness, rash, drowsiness, etc. (Table 2).

\section{Seizure Frequency and Change Overtime}

The minimum and the maximum number of measurements for the number of seizure attacks per individual were 2 and 8 respectively. While the average number of measurements, $i$. e., the average repetitive times that the number of seizure attacks recorded was 5.5. Over the follow-up period, patients had a minimum of 1 and a maximum of 30 seizure attacks. The median baseline number of seizure attacks among patients was 3 times with the interquartile range (IQR) of 2 and it showed a quick decline over time. In addition, the average number of seizure attacks at baseline was 3.57 times/patient with an SD of 2.75 times/patient (Table 3).

\section{Exploratory Data Analysis of Seizure Frequency Overtime}

The individual profile plot for a sample of 100 subjects was done. From the panel we noted that there is a considerably large difference in the intercepts of individual trajectories and similarly, some trajectories were steeper while others were almost horizontal, indicating the possible variability in the slope of the number of seizure attacks (Figure 1).

The subjects have a largely varying number of seizure attacks at the start and also possibly different evolutions over time; this suggests that perhaps generalized linear mixed models with random intercepts and slopes could be a plausible starting point. The overall mean profile plot shows that approximately a linearly decreasing pattern of the number of seizure attacks over time even if it increases at some time points which suggests that a linear time effect is reasonable (Figure 2). 
Table 2 Clinical and Treatment Related Characteristics of AED Attendants, Amanuel Mental Specialized Hospital, 2017-2019

\begin{tabular}{|c|c|}
\hline Variables with Category & $\begin{array}{l}\text { Epilepsy Cases Among Each } \\
\text { Category }(n=394)(\%)\end{array}$ \\
\hline Head Injury & \\
\hline Yes & $62(15.74)$ \\
\hline No & $332(84.26)$ \\
\hline Burn & \\
\hline Yes & $15(3.81)$ \\
\hline No & $379(96.19)$ \\
\hline Fracture & \\
\hline Yes & $9(2.28)$ \\
\hline No & $385(97.72)$ \\
\hline Depression & \\
\hline Yes & $49(12.44)$ \\
\hline No & $345(87.56)$ \\
\hline Psychotic disorder & \\
\hline Yes & $66(16.75)$ \\
\hline No & $328(83.25)$ \\
\hline Anxiety & \\
\hline Yes & $32(8.12)$ \\
\hline No & $362(91.88)$ \\
\hline $\begin{array}{l}\text { Type of treatment when } \\
\text { treatment started }\end{array}$ & \\
\hline Phenobarbital & $203(51.52)$ \\
\hline Carbamazepine & $49(12.44)$ \\
\hline Phenytoin & $29(7.36)$ \\
\hline Sodium-Valporate & $52(13.20)$ \\
\hline Two or more treatment & $61(15.48)$ \\
\hline Treatment Modality & \\
\hline Monotherapy & $293(74.37)$ \\
\hline Polytherapy & $101(25.63)$ \\
\hline Treatment related side effects & \\
\hline Yes & $34(8.63)$ \\
\hline No & $360(91.37)$ \\
\hline Type of Seizure during diagnosis & \\
\hline Partial & $27(6.80)$ \\
\hline Generalized & $252(64.00)$ \\
\hline Unclassified & $115(29.20)$ \\
\hline
\end{tabular}

\section{Factors Influencing Seizure Frequency}

The response variable for the study was the number of seizure attacks among epileptic patients which is a count variable and recorded repeatedly per person. An initial assumed model for count longitudinal data is a GLMM for the Poisson family which assumes that the mean is equal to variance but for this particular data this
Table 3 Descriptive Statistics for the Number of Seizure Attack per Follow-Up Time

\begin{tabular}{|l|l|l|l|l|}
\hline $\begin{array}{l}\text { Follow-Up Time } \\
\text { (Months) }\end{array}$ & Median & Mean & Std.Dev & $\mathbf{N}$ \\
\hline Baseline & 3 & 3.56 & 2.75 & 394 \\
1 & 1 & 1.73 & 2.12 & 394 \\
2 & 1 & 1.26 & 1.76 & 383 \\
3 & 0 & 1.08 & 1.65 & 341 \\
4 & 0 & 0.97 & 1.70 & 269 \\
5 & 0 & 0.79 & 1.40 & 191 \\
6 & 0 & 1.05 & 1.72 & 118 \\
7 & 1 & 1.50 & 2.00 & 58 \\
\hline
\end{tabular}

assumption is violated which was checked by using package DHARMa on $\mathrm{R}$ and implies that there is overdispersion in this particular data. In order, to handle this over-dispersion a GLMM for the negative binomial family was considered and from the model output, the dispersion parameter was found to be 2.69 with a p-value $<0.0001$. Regarding the zero-inflation factor Vuong test was done and it favors a GLMM for the negative binomial model over a GLMM for the zero-inflated negative binomial family. Therefore, a final model used in this study is a GLMM for the negative binomial family building on function glmer on R-software.

Firstly, we used a stepwise automatic variable selection method (backward elimination method) to select appropriate fixed-effects. After arriving at an optimal subset, the selected fixed-effects model was fitted with different random effects to have an appropriate generalized linear mixed-effect model (GLMM). Finally, when we look at the improvement of the model with the inclusion of random intercept to that of random linear time effects of the GLMM, there was an improvement of the model and this model has lower AIC and BIC values than the remaining two GLMMs. Hence, we consider the GLMM with random intercept and linear time effects as a better parsimonious model (Table 4).

It was found that keeping all other variables constant, for each additional three months, the number of seizure attacks would decrease on average by $16 \%$ (AIRR $=0.84$ and $95 \% \mathrm{CI}: 0.820 .85$ ). Conditional on the random effects the incidence rate of seizure attack was decreased by $19 \%$ $(\mathrm{AIRR}=0.81$ and $95 \% \mathrm{CI}: 0.690 .94)$ among the age group of 25-34 as compared to those who are in the age group of 15-24. Conversely, conditional on the random effects the incidence rate of seizure attacks among those who smoked was increased by $28 \%$ (AIRR $=1.28$ and $95 \%$ 


\section{Individual Profile Plot for 100 Subjects}

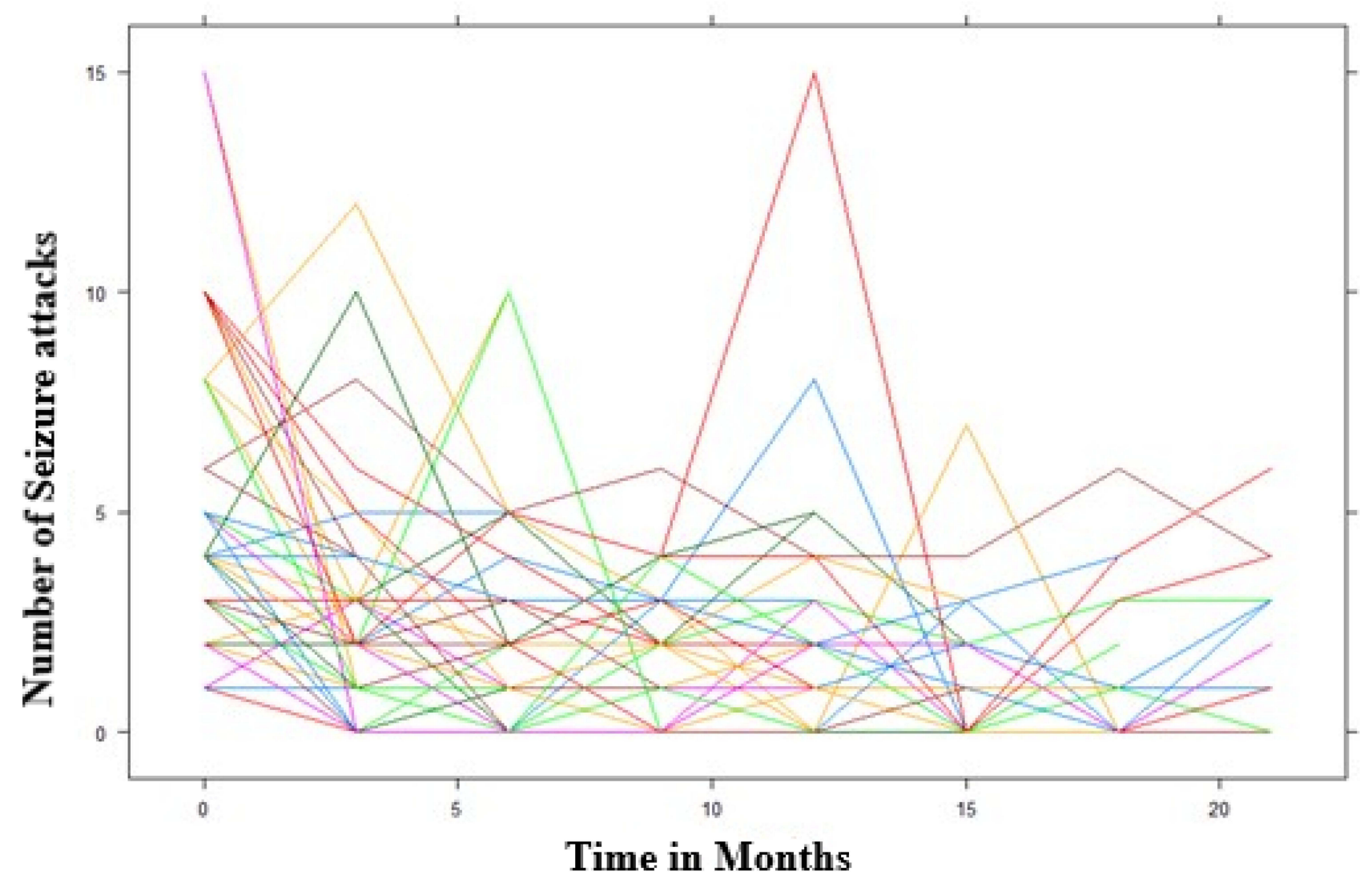

Figure I Individual profile plot for the number of seizure attacks.

CI: 1.11 1.48) as compared to those who do not. Conditional on the random effects, the incidence rate of seizure attacks among those who have experienced sleep deprivation was increased by $49 \%$ (AIRR $=1.49$ and $95 \%$ CI: 1.32 1.69).

Conditional on the random effects those patients who have a history of depression were found to be a 1.39 times more incidence rate of seizure attacks than those who do not have a history of depression (AIRR $=1.39$ and $95 \%$ CI: 1.17 1.65). Conditional on the random effects the incidence rate of seizure attack among those who take polytherapy was increased by $22 \%($ AIRR $=1.22$ and 95\% CI: 1.071 .39 ) as compared to those who take monotherapy (Table 5).

\section{Discussion}

From an individual's profile plot, we observed the existence of variability in the number of seizure attacks within and between individuals. The exploratory analysis result for mean structure also suggested that on average, the number of seizure attacks decreases in a linear pattern over time. This supports the results of who found that after the patients initiated to the AEDs the number of seizure attacks decreased due to the therapy even if some patients are refractory to any AEDs. ${ }^{10}$ This linear decrease in the number of seizure attacks is the positive effect of treatment on controlling seizures. Besides, the finding showed good control of seizure attacks through therapies. However, an increase of the mean profile after some time might be associated with AED dose tapering that might decrease serum level that leads to a resurgence of seizure frequencies. Also, as the duration of the treatment increased patients may have become exhausted from adverse drug effects and that could compromise the adherence of the patients to their AED medication.

From the Multivariable GLMM, we found that covariates that have a significant effect on the number of seizure attacks change, those covariates are observation time, age, smoking, khat use, sleep deprivation, head injury, depression, clumsiness, and treatment mode.

This study found that a negative significant association between observation time and seizure attacks, which 


\section{The Mean Profile}

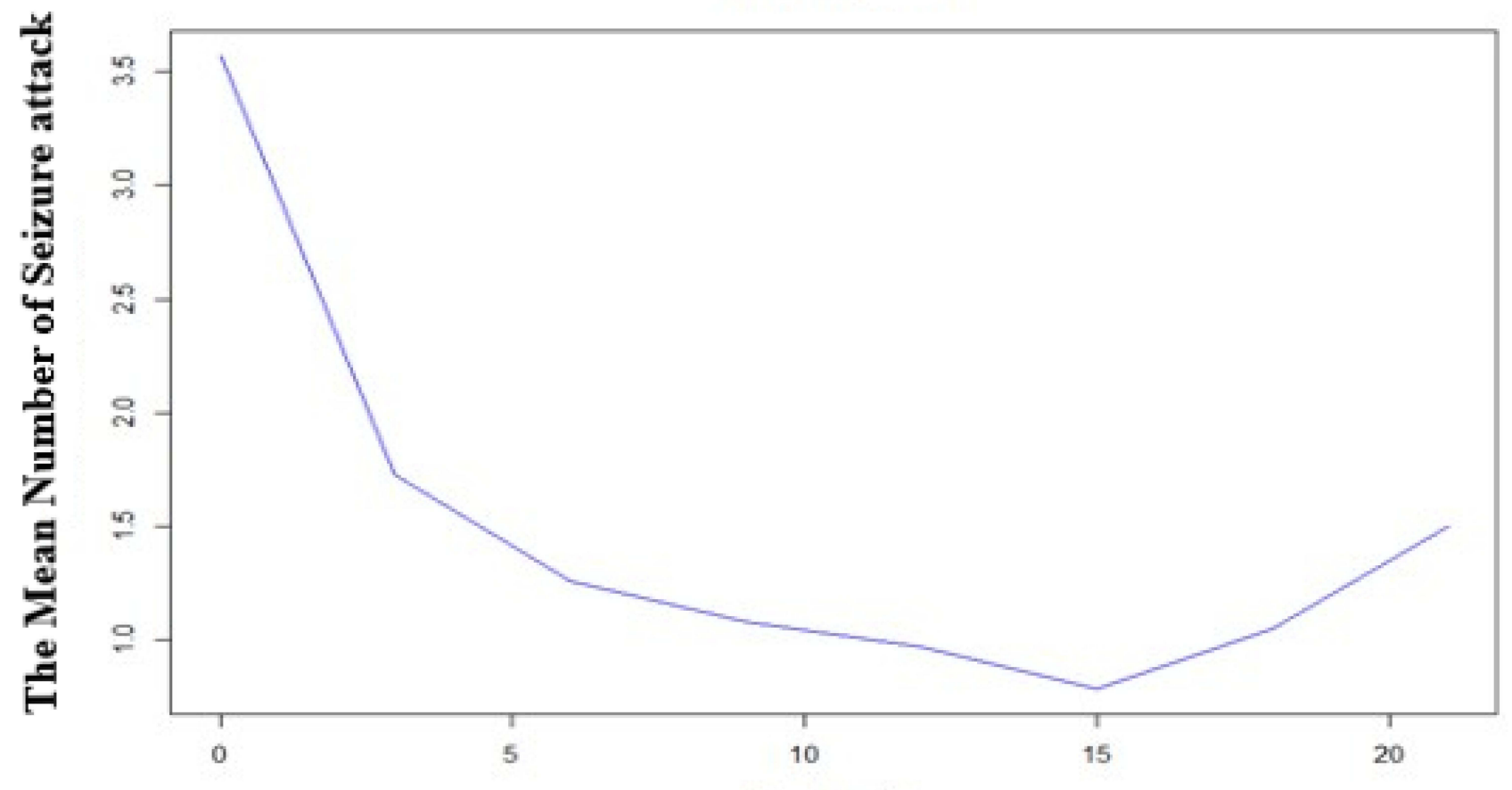

\section{Time in Months}

Figure 2 Mean profile plot for the number of seizure attacks.

means that as an observation time increases the number of seizure attacks would decrease. This is in line with the study conducted in Bahir Dar. ${ }^{11}$ This indicates a positive treatment outcome on controlling seizures. However, this study differs from a study conducted in Sweden. ${ }^{10}$ This could be because, some patients tend to be stable or "spontaneously" get poor, which could be attributed to poor compliance with their current medications. The other possible reason for this might be the inclusion of those patients who have SE in the other studies.

This study found an association between age and seizure attacks, indicating that patients in the age group of 25-34 had a lower incidence rate of seizure attack as compared to those who were in the age group of 15-24. This was in line with a previous study. ${ }^{12}$ This differed from a study conducted in the United States, which

Table 4 Selection of Random Effects to be Included in the GLMM

\begin{tabular}{|l|l|r|r|l|}
\hline No. & \multicolumn{1}{|c|}{ Random Effects } & AIC & BIC & Deviance \\
\hline I. & Only intercept & 6815.2 & 6900.3 & 6785.2 \\
2. & Only linear time slope & 6572.4 & 6657.4 & 6542.4 \\
3. & Intercept and linear time slope & 6564.0 & 6654.7 & 6532.0 \\
\hline
\end{tabular}

demonstrated no significant correlation between the changes in the seizure frequency from the baseline to the end of the follow-up period and age. ${ }^{13}$ This could be because patients aged 25-34 years may have good adherence compared to those aged 15-24 years. ${ }^{14}$ However, no consistency was found between the cited and the current study concerning patients' age.

Substance use, such as a history of smoking and chewing khat, were found to have more frequent seizure attacks compared to those who had no such history. This is in line with the study conducted in Ayder comprehensive hospital. ${ }^{15}$ This could be explained that chewing khat and smoking are related to generalized tonic-clonic seizures, and they may progress into SE. ${ }^{16}$ Besides, substance use causes less adherent to medications. Hence, substance abuse needs adequate management to improve the hospital outcomes of epilepsy patients.

This study found a positive significant association between sleep deprivation and seizure attack. Respondents who reported sleep deprivation had a higher incidence rate of seizure attack as compared to those who do not report. This is in line with a multi-center study conducted in Denmark, Norway, and United States which reports that patients with generalized epilepsy were found to be highly 
Table 5 Risk Factors for Seizure Attacks Among Adult Epilepsy Patients at $\mathrm{AMSH}$

\begin{tabular}{|c|c|c|}
\hline $\begin{array}{l}\text { Variables with } \\
\text { Category }\end{array}$ & CIRR(95\% CI) & $\operatorname{AIRR}(95 \% \mathrm{CI})$ \\
\hline Time & $0.83(0.82-0.85)^{* * *}$ & $0.84(0.82-0.85)^{* * *}$ \\
\hline Age & & \\
\hline $15-24$ & 1 & I \\
\hline $25-34$ & $0.985(0.98-0.99)^{* * *}$ & $0.8 \mathrm{I}(0.69-0.94)^{* *}$ \\
\hline $35-44$ & $1.014(1.013-1.015)^{* * * *}$ & $0.94(0.78-1.13)$ \\
\hline $45-54$ & $1.002(1.001-1.003)^{* * *}$ & $0.7 \mid(0.48-1.04)$ \\
\hline $55-65$ & $1.181(1.180-1.182)^{* * * *}$ & $0.94(0.69-1.27)$ \\
\hline Smoking & & \\
\hline No & 1 & I \\
\hline Yes & $1.54(I .3 \mathrm{I}-\mathrm{I} .8 \mathrm{I})^{* * * *}$ & $1.28(1.11-1.48)^{* *}$ \\
\hline Khat & & \\
\hline No & I & I \\
\hline Yes & $1.69(1.44-1.99)^{* * * *}$ & $1.39(1.19-1.62)^{* * *}$ \\
\hline Sleep deprivation & & \\
\hline No & I & 1 \\
\hline Yes & $1.66(1.45-1.91)^{* * *}$ & $1.49(1.32-1.69)^{* * * *}$ \\
\hline Head injury & & \\
\hline No & 1 & 1 \\
\hline Yes & I.7I $(1.44-2.04)^{* * *}$ & $1.30(1.11-1.53)^{* *}$ \\
\hline Depression & & \\
\hline No & I & 1 \\
\hline Yes & $1.63(1.34-1.98)^{* * * *}$ & $1.39(1.17-1.65)^{* * *}$ \\
\hline Treatment & & \\
\hline Modality & & \\
\hline Monotherapy & 1 & 1 \\
\hline Polytherapy & $1.27(1.08-1.49)^{* *}$ & $1.22(1.07-1.39)^{* *}$ \\
\hline Clumsiness & & \\
\hline No & 1 & 1 \\
\hline Yes & $2.59(1.65-4.10)^{* * * *}$ & $1.85(1.24-2.75)^{* *}$ \\
\hline
\end{tabular}

Notes: ${ }^{* * *}$ Implies $\mathrm{p}$-value $<0.0000 \mathrm{I}$ and $*^{*}$ implies $\mathrm{p}$-value $<0.0 \mathrm{I}$.

sensitive to sleep deprivation and seizure attacks. ${ }^{17}$ The possible reason could be the majority of the respondents in the present study diagnosed with epilepsy were with generalized seizure type. Sleep pattern disorder has been shown to lower the seizure threshold, doing so makes it easy to provoke a seizure attack. ${ }^{18}$ However, the same study showed no statistically significant association between sleep deprivation and seizure attack. ${ }^{19}$ This may be because the study was limited to patients with refractory epilepsy and the participants did not consume any illicit drug. However, in the present study, some respondents consume alcohol, cigarette, and chat.
Participants who experienced head injury were more likely to had more seizure attacks as compared to those who did not experience a head injury. This finding is in line with that of Addis Ababa and Gondar. ${ }^{20-22}$ This may be explained by the head injury that may cause the deposit of iron associated with extravasation of blood, the increase in excitotoxicity due to the accumulation of glutamate, and diffuse axonal injury edema or ischemia. ${ }^{23}$

In this study, depression was found to have a statistically significant positive association with seizure attacks. This is supported by the study conducted in Ethiopia. ${ }^{24}$ The possible reason could be that depression seems to affect the quality of life of epileptic patients and this leads to a poorer treatment outcome. ${ }^{16}$ Thus, clinicians need to focus more on comorbid depression to improve treatment outcomes.

This study found a positive statistically significant association between treatment modality and seizure attack. Respondents who have taken two or more treatments at a time had a higher incidence rate of seizure attack as compared to those who have taken only one treatment. This is supported by different studies..$^{13,24,25}$ The possible reason could be Polytherapy increases the potential for drug-drug interactions, may affect compliance and is associated with a higher cost of medication requirement of therapeutic drug monitoring. ${ }^{26}$ In addition, those patients who were resistant to Monotherapy featured by high-frequency seizure attacks are eligible for Polytherapy. Thus, patients who were on Polytherapy had a high frequency of seizure attacks compared to those on Monotherapy.

This study found a positive statistical association between the side effects of medications and seizure attacks. People who had side effects of medication like that of clumsiness were found to have frequent seizure attacks as compared to people who had no side effects of medications. This is in line with the study. ${ }^{24}$ This might be due to the patients in this study were taken a Polytherapy and have psychiatric comorbidities which both expose the patients to treatment-related side effects.

This study has an implication for improvement in the health care practice of health professionals while providing care for patients with epilepsy including improvement in communication between health professionals and patients with epilepsy. The other implication of this study goes to decision-makers to consider epilepsy care-related programs and policy. This study also indicates the need for a future prospective study. 


\section{Limitations}

The main limitations of this study are since the study was based on record review and data was not available for some relevant variables (e.g., Cause of epilepsy, cost of medication, Adherence to medication, mode of diagnosis, and baseline clinical factors like a bacterial infection, viral infection, etc.), the effect of such unmeasured variables was not estimated separately. Some of the variables used like depression were not measured we took the only diagnosis from the patient medical follow-up sheets. In addition, some of the studies used for discussion have a very small sample size which makes the comparison difficult.

\section{Conclusion}

The number of seizure attack changes over time could be sufficiently described by a declining linear function, which implies AED were controlling the seizure frequencies. The number of seizure attacks was relatively high at the baseline. The influences of several covariates on the evolution of the number of seizure attacks were identified. Among this age, smoking, khat use, sleep deprivation, head injury, depression, clumsiness, and treatment modality was the determinant factors that determine the change in the number of seizure attack in this study. Results revealed that a reduction in the number of AEDs used led to a considerable improvement in seizure control. We recommend future researchers conduct a multi-center prospective study by including other essential covariates that were not included in this study (e.g., Cause of epilepsy, cost of medication, adherence to medication, mode of diagnosis, and baseline clinical factors like a bacterial infection, viral infection, etc.).

\section{Abbreviations}

AED, anti-epileptic drug; AIC, Akaike Information Criteria; AIRR, Adjusted Incidence Rate Ratio; AMSH, Amanuel Mental Specialized Hospital; BIC, Bayesian Information Criteria; CI, Confidence Interval; CIRR, Crude Incidence Rate Ratio; CNS, Central Nervous System; EEG, electroencephalographic; GLMM, Generalized Linear Mixed Model; GTCS, generalized tonic-clonic seizures; IQR, interquartile range; LMIC, low and middle-income countries; LRT, Likelihood Ratio Test; NB, negative binomial; NEP, Neuropsychiatric; SD, standard deviation; SE, Status Epilepticus.

\section{Data Sharing Statement}

The dataset analyzed during the current study available from the corresponding author on reasonable request.

\section{Acknowledgments}

We would like to thanks all data collectors and study participants for their cooperation and support during the study period.

\section{Author Contributions}

All authors made a significant contribution to the work reported, whether that is in the conception, study design, execution, acquisition of data, analysis, and interpretation, or in all these areas; took part in drafting, revising or critically reviewing the article; gave final approval of the version to be published; have agreed on the journal to which the article has been submitted; and agree to be accountable for all aspects of the work.

\section{Funding}

This work was supported financially by the University of Gondar. However, the University had no role in study design, data collections, and analysis, decision to publish, or preparation of the manuscript.

\section{Disclosure}

The authors report no conflicts of interest related to this work.

\section{References}

1. Keezer MR, Sander JW, Sander JW. Comorbidities of epilepsy: current concepts and future perspectives. Lancet Neurol. 2016;15(1):106-115. doi:10.1016/S1474-4422(15)00225-2

2. Feigin VL, Abajobir AA, Abate KH. Global, regional, and national burden of neurological disorders during 1990-2015: a systematic analysis for the Global Burden of Disease Study 2015. Lancet Neurol. 2017;16(11):877-897. doi:10.1016/S1474-4422(17)30299-5

3. Chen Z, Liew D, Liew D, et al. Treatment outcomes in patients with newly diagnosed epilepsy treated with established and new antiepileptic drugs: a 30-year longitudinal cohort study. JAMA Neurol. 2018;75 (3):279-286. doi:10.1001/jamaneurol.2017.3949

4. Nabukenya AM, Matovu JK, Wabwire-Mangen F, Wanyenze RK, Makumbi F. Health-related quality of life in epilepsy patients receiving anti-epileptic drugs at National Referral Hospitals in Uganda: a cross-sectional study. Health Qual Life Outcomes. 2014;12:49. doi:10.1186/1477-7525-12-49

5. Bifftu BB, Dachew BA, Tiruneh BT. Perceived stigma and associated factors among people with epilepsy at Gondar University Hospital, Northwest Ethiopia: a cross-sectional institution based study. Afr Health Sci. 2015;15(4):1211-1219. doi:10.4314/ahs.v15i4.21

6. Hasiso TY, Desse TA, Romigi A. Adherence to treatment and factors affecting adherence of epileptic patients at Yirgalem General Hospital, Southern Ethiopia: a prospective cross-sectional study. PLoS One. 2016;11(9):e0163040. doi:10.1371/journal.pone.0163040 
7. Amare A, Zenebe G, Hammack J, Davey G. Status epilepticus: clinical presentation, cause, outcome, and predictors of death in 119 Ethiopian patients. Epilepsia. 2008;49(4):600-607. doi:10.1111/ j.1528-1167.2008.01556.x

8. Diggle P, Heagerty P, Liang K-Y, Zeger S. Analysis of Longitudinal Data. 2nd ed. New York: Oxford University Press; 2002.

9. Foundation AaD. Khat. 2021.

10. Ahn JE, Plan EL, Karlsson MO, Miller R. Modeling longitudinal daily seizure frequency data from pregabalin add-on treatment. J Clin Pharmacol. 2012;52(6):880-892. doi:10.1177/0091270011407193

11. Mekonnen FH, Tesfaye ZD, Swain PK, Swain PK. Statistical models for longitudinal zero-inflated count data: application to seizure attacks. African Health Sci. 2019;19(3):2555-2564. doi:10.4314/ ahs.v19i3.31

12. Banerjee PN, Filippi D, Hauser WA. The descriptive epidemiology of epilepsy-a review. NIH Public Access. 2009;85(1):31-45.

13. Salih MR, Bahari MB, Hassali MA, et al.Characteristics of seizure frequency among Malaysian children diagnosed with structuralmetabolic epilepsy. J Neurosci Rural Pract. 2012;3(3):244-250.

14. Dworetzky BA, Bromfield EB, Townsend MK, Kang JH. A prospective study of smoking, caffeine, and alcohol as risk factors for seizures or epilepsy in young adult women: data from the Nurses' Health Study II. Epilepsia. 2010;51(2):195-205.

15. Niriayo YL, Mamo A, Kassa TD, et al. Treatment outcome and associated factors among patients with epilepsy. Sci Rep. 2018;8:17354. doi:10.1038/s41598-018-35906-2

16. Patel RS, Mansuri Z, Elmaadawi A, Kaur M, Shah K, Nasr S. Psychiatric comorbidities and outcomes in epilepsy patients: an insight from a nationwide inpatient analysis in the United States. Cureus. 2017;9(9):e1686.

17. Nakken KO, Solaas MH, Kjeldsen MJ, Friis ML, Pellock JM, Corey LA. Which seizure-precipitating factors do patients with epilepsy most frequently report? Epilepsy Behav. 2005;6:85-89. doi:10.1016/j.yebeh.2004.11.003
18. Sanya E, Mustapha K, Ademiloyi A, Bello A, Alaofin O. Self-perceived seizure precipitants among patients with epilepsy in middle-belt Nigeria. Niger J Clin Pract. 2014;17(6):701-705. doi:10.4103/1119-3077.144381

19. Malow BA, Passaro E, Milling C, Minecan DN, Levy K. Sleep deprivation does not affect seizure frequency during inpatient video-EEG monitoring. Neurology. 2002;59:1371-1374. doi:10.1212/01.WNL.0000031810.15811.9E

20. Tigistu M, Azale T, Kebebe H, Yihunie T. Frequency of seizure attack and associated factors among patients with epilepsy at University of Gondar Referral Hospital: a cross-sectional study, Gondar, North West Ethiopia, 2017. BMC Res Notes. 2018;11 (1):652. doi:10.1186/s13104-018-3761-3

21. Fanta T, Azale T, Assefa D, Getachew M. Prevalence and factors associated with perceived stigma among patients with epilepsy in Ethiopia. Psychiatry J. 2015;2015:627345. doi:10.1155/2015/627345

22. Lowenstein DH. Epilepsy after head injury: an overview. Epilepsia. 2009;50(2):4-9. doi:10.1111/j.1528-1167.2008.02004.x

23. Tellez-Zenteno JF, Patten SB, Jetté N, Williams J, Wiebe S. Psychiatric comorbidity in epilepsy: a population-based analysis. Epilepsia. 2007;48:2336-2344.

24. Tegegne MT, Mossie TB, Awoke AA, Assaye AM, Gebrie BT, Eshetu DA. Depression and anxiety disorder among epileptic people at Amanuel Specialized Mental Hospital, Addis Ababa, Ethiopia. BMC Psychiatry. 2015;15:210. doi:10.1186/s12888-015-0589-4

25. Afifi L, Al-Kattan M, Mostafa ED, Shamloul R. Assessment of precipitating factors of breakthrough seizures in epileptic patients. Egypt J Neurol Psychiatr Neurosurg. 2015;52(3):165-171. doi:10.4103/1110-1083.162002

26. Wahab A. Difficulties in treatment and management of epilepsy and challenges in new drug development. Pharmaceuticals (Basel). 2010;3(7):2090-2110. doi:10.3390/ph3072090
Neuropsychiatric Disease and Treatment

\section{Publish your work in this journal}

Neuropsychiatric Disease and Treatment is an international, peerreviewed journal of clinical therapeutics and pharmacology focusing on concise rapid reporting of clinical or pre-clinical studies on a range of neuropsychiatric and neurological disorders. This journal is indexed on PubMed Central, the 'PsycINFO' database and CAS, and is the official journal of The International Neuropsychiatric Association (INA). The manuscript management system is completely online and includes a very quick and fair peer-review system, which is all easy to use. Visit http://www.dovepress.com/testimonials.php to read real quotes from published authors. 